\title{
Editorial
}

\section{Advances in Antenna Technology for Wireless Handheld Devices}

\author{
Jaume Anguera, ${ }^{1,2}$ Aurora Andújar, ${ }^{1}$ Minh-Chau Huynh, ${ }^{3,4}$ and Charlie Orlenius ${ }^{5}$ \\ ${ }^{1}$ Technology and Intellectual Property Rights Department, Fractus, Barcelona, Spain \\ ${ }^{2}$ Electronics and Communications Department, Ramon Llull University, Barcelona, Spain \\ ${ }^{3}$ Systems and Concept, Sony Mobile, Redwood City, CA, USA \\ ${ }^{4}$ Communications Systems Group, LitePoint Corporation, CA, USA \\ ${ }^{5}$ Bluetest $A B$, Gothenburg, Sweden \\ Correspondence should be addressed to Jaume Anguera; jaume.anguera@fractus.com
}

Received 16 December 2012; Accepted 16 December 2012

Copyright (C) 2013 Jaume Anguera et al. This is an open access article distributed under the Creative Commons Attribution License, which permits unrestricted use, distribution, and reproduction in any medium, provided the original work is properly cited.

Communication between two distant points has been a constant challenge for mankind, from ancient smoke signals, to telegraph, to finally wireless communication through electromagnetic signals. This evolution represents a constant effort to improve the quality and effectiveness of distance communication with ever-evolving techniques to enhance the delivery of contents, from voice to data. Wireless handheld devices are the most representative paradigm of these efforts. Since they first appeared, their size has continuously been shrinking, while their functional capabilities have been increasing, hence creating the never-ending challenge in antenna design. In this regard, the antenna community often has the important role of designing low-profile, small, and multiband antennas capable of being integrated within the handset platform as well as capable to coexist with multiple antenna systems in order to satisfy the strict demands of emergent multifunction wireless devices. Furthermore, the complexity of handheld antenna design is continuously increasing, not only by the pressure of the market needs, but also by the duty of safety regulations which require efficient antennas capable of radiating as much power as possible in free-space conditions, while minimizing the power radiated towards the human head.

Antenna modeling in handset devices, using electromagnetic simulation software, has improved significantly thanks to the progress of computing hardware. Complex environments surrounding the antenna, such as a handset device held beside a human head, and precise details on nearby components, including the presence of a loudspeaker in the antenna volume, can be modeled accurately to predict antenna performance that is closer to reality, without sacrificing simulation speed. This evolution considerably contributes to simplify the antenna design process.

Not only the simulation tools have considerably evolved in the latest years, but also the measurement systems have been forced to evolve for satisfying the emergent communication systems requirements. These recent advances in measurement systems and methodologies have been hot topics in the antenna measurement community for capturing, for instance, radiated performance in the emergent LTE and MIMO antenna systems. These next generation systems have already started to appear in wireless handheld devices in the consumer market. However, new measurement methods need to be developed as these antenna systems are to be used as well as tested in fading environments.

Finally, the commercial success of wireless handheld devices leads to an improvement of the manufacturing techniques and processes. This is not only important for reducing the cost of mass production, but also for enhancing the design performance and size in a controlled fashion.

This special issue contains five papers that gather some of the recent advancements in handset antenna design. In the paper entitled "Band-notched ultrawide band planar inverted$F$ antenna," an ultrawide planar inverted-F antenna covering the $3.4 \mathrm{GHz}-11.2 \mathrm{GHz}$ band with a band-notch at $5.08 \mathrm{GHz}-$ $6 \mathrm{GHz}$ is presented. The wideband behavior is obtained by parasitic elements whereas the band-notch is achieved by a $\mathrm{W}$-shaped slot on the top radiating element of the antenna. 
The paper "Compact dual-band dual-polarized antenna for MIMO LTE applications" proposes an antenna system operating in the LTE bands $700 \mathrm{MHz}-862 \mathrm{MHz}$ and $2.5 \mathrm{GHz}-2.69 \mathrm{GHz}$. The design is composed of two compact orthogonal monopoles to perform diversity in mobile terminals such as tablets or laptops.

In the paper, "Printed internal pentaband WWAN antenna using chip-inductor-loaded shorting strip for mobile phone application", a compact size on-Board printed antenna using capacitive coupled-fed excitation to generate multiple resonant modes for pentaband WWAN operation (GSM850/900, GSM1800/1900, UMTS2100) is designed.

The paper "Evaluation of SAR distribution in six-layer human head model" numerically analyzes a single layer and a six-layer human head model for SAR computation at the $900 \mathrm{MHz}$ frequency.

Finally, in the paper "Advances in antenna technology for wireless handheld devices", the evolution of wireless handheld devices, regulations, and challenges in today's smartphones, and handset characterization are reviewed. Finally, recent advancements in antenna technology for wireless handheld or portable devices are presented.

Jaume Anguera Aurora Andújar Minh-Chau Huynh

Charlie Orlenius 



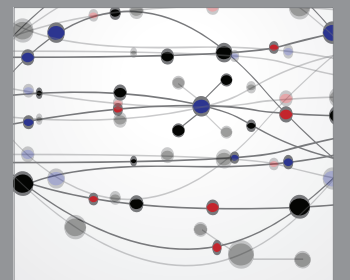

The Scientific World Journal
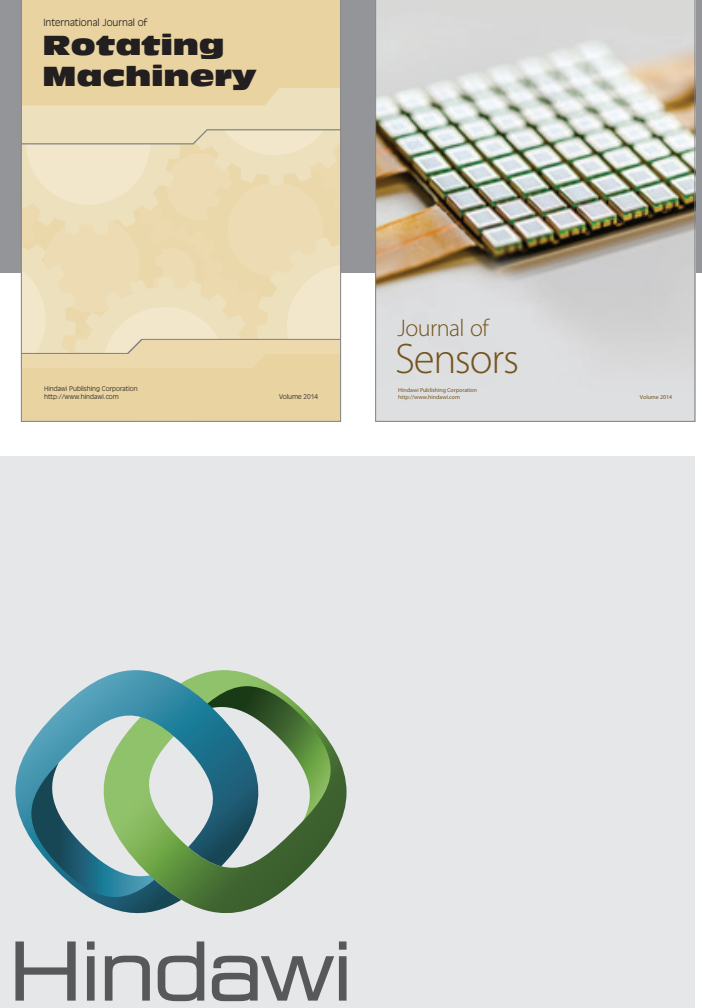

Submit your manuscripts at http://www.hindawi.com
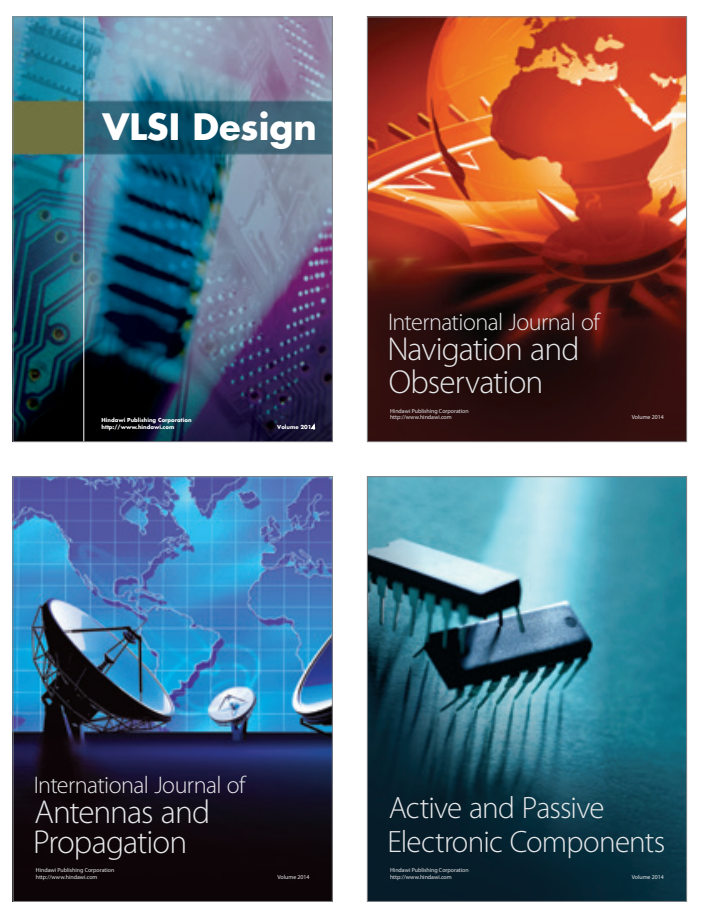
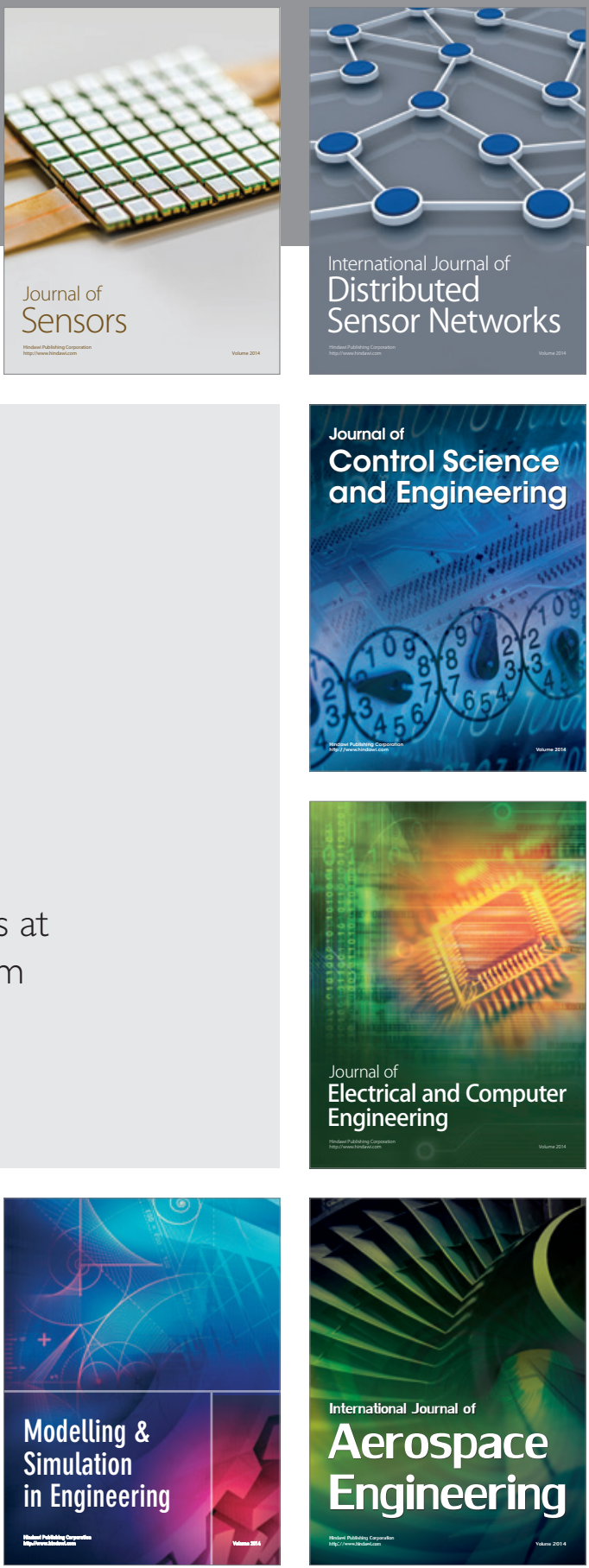

Journal of

Control Science

and Engineering
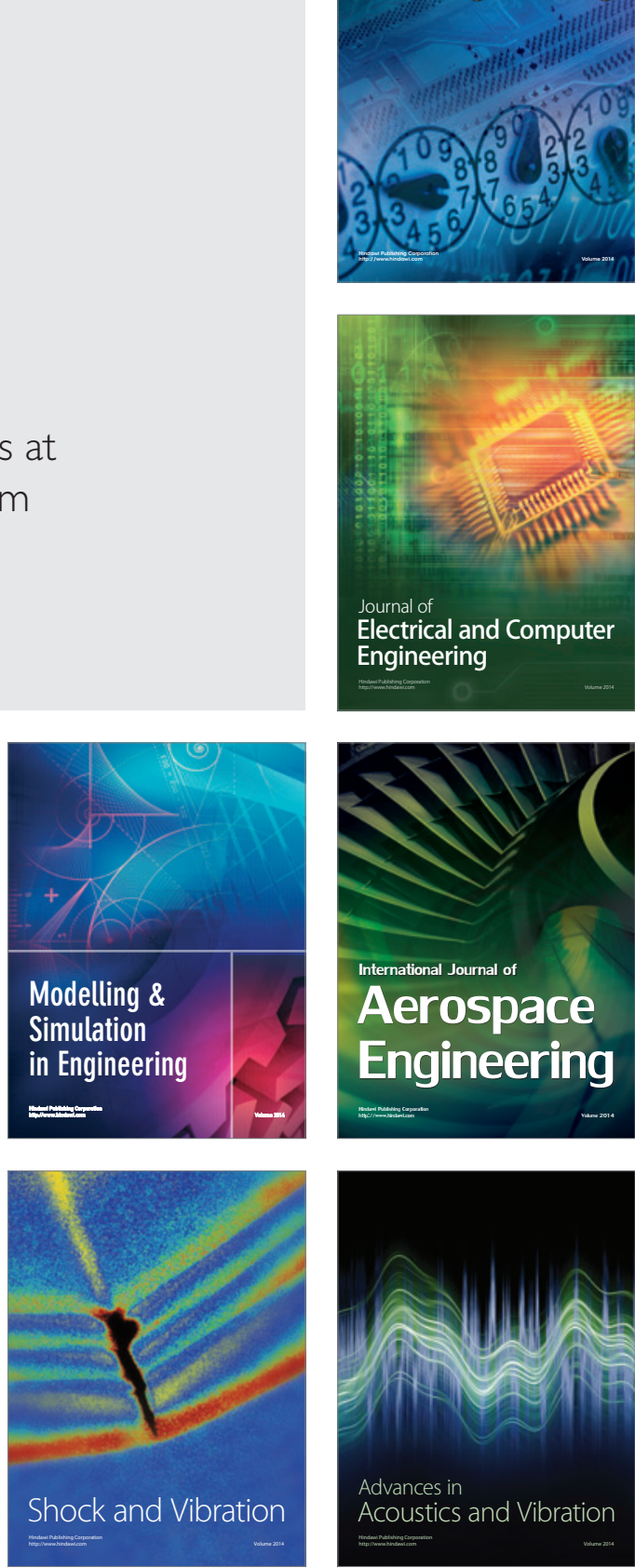